\title{
Validation of the Flow Topology Around Several Airdrop Cargo Configurations at Static Conditions
}

\author{
Sven Geisbauer* Niko Bier, Jochen Kirz ${ }^{\dagger}$ \\ German Aerospace Center (DLR), 38108 Braunschweig, Germany \\ and \\ Eric W. M. Roosenboom ${ }^{\ddagger}$ \\ German Aerospace Center (DLR), 37073 Göttingen, Germany
}

\begin{abstract}
A numerical study was carried out to assess the interference effects between the wake of a transport aircraft and several generic cargo bodies during the early stage of an airdrop scenario. DLR carried out extensive wind tunnel airdrop investigations between 2006 and 2012. Based on the experimental data and preceding numerical simulations distinct positions of the trajectory being subject to strong interference effects were statically reproduced. The flow field around the bodies was experimentally investigated using stereoscopic Particle Image Velocimetry and compared to steady and unsteady Reynoldsaveraged Navier-Stokes (RANS) computations. The latter were carried out using the unstructured DLR TAU code. The primary focus is to assess the suitability, accuracy and the limitations of RANS methods in such challenging flow conditions. Therefore, the influence of several turbulence models was investigated and compared to experimental field velocity data. In addition, an exemplary unsteady RANS simulation was conducted to highlight the differences to the steady approach. Although deviations in the wake of the bodies were observed, the qualitative agreement between the steady simulations and experiment was very good. Quantitatively, however, the steady approach leaves room for further improvements. The gap to the experimental data could partially be reduced in applying unsteady RANS methods.
\end{abstract}

\section{Nomenclature}

$\begin{array}{ll}\text { DLR } & \text { Deutsches Zentrum fuer Luft- und Raumfahrt (German Aerospace Center) } \\ \text { SSG/LRR- } \omega & \text { Speziale-Sarkar-Gatski/Launder-Reece-Rodi (Reynolds stress model) } \\ C_{D} & \text { Drag coefficient } \\ C_{L} & \text { Lift coefficient } \\ U_{\infty} & \text { Free stream velocity } \\ V & \text { Magnitude of velocity vector } \\ u, v, w & \text { Velocity components in } x, y \text { and } z \text {-directions } \\ x, y, z & \text { Cartesian axis system }\end{array}$

\section{Introduction}

$A^{N}$ accurate simulation of the motion of airdropped cargo with Computational Fluid Dynamics (CFD) Amethods requires a profound knowledge and resolution of the prevailing flow features. The flow behind

*Research Engineer, Transport Aircraft Department, Institute of Aerodynamics and Flow Technology, Lilienthalplatz 7.

${ }^{\dagger}$ Bachelor Student, Transport Aircraft Department, Institute of Aerodynamics and Flow Technology, Lilienthalplatz 7.

${ }^{\ddagger}$ Research Scientist, Experimental Methods Department, Institute of Aerodynamics and Flow Technology, Bunsenstr. 10. AIAA Senior member. 
modern military transport aircraft typically is highly vortical due to their geometric shape, in particular when the ramp is lowered during flight ${ }^{1}$. Very lightweight or aerodynamically sensitive cargo, or paratroopers, are susceptible to the local flow conditions and prone to colliding with the hull ${ }^{2,3}$. Consequently, if the motion of the cargo was known in advance the safety of the aircraft, the crew and the cargo could be significantly improved.

In recent years, the progress in numerical tools allowed for a more accurate simulation of the flow field behind the aircraft ${ }^{4,5}$ or about cargo configurations ${ }^{6,7}$. Increasing efforts have been taken to apply highfidelity CFD methods to simulate near-field airdrop trajectories ${ }^{8-10}$. In addition, extensive experimental test campaigns, investigating the flow field and the acting forces during airdrop operations, were carried out, mainly to provide validation data ${ }^{11-13}$.

Within the internal projects MiTraPor (2006-2009) and MiTraPor II (2010-2013) DLR has committed to progressively enhance its capabilities in evaluating the performance of military transport aircraft. Unlike their civil counterparts military transport aircraft need to perform safely in a much wider range of applications. Operational scenarios such as tactical low-altitude flight, flying in close formation or airdrop delivery are just a few examples. The performance assessment of military transport aircraft in their operational environment therefore requires dedicated evaluation tools.

As of 2006, DLR has begun to systematically extend its competence in assessing airdrop operations. The challenge still consists in accurately predicting the trajectory and time-dependent motion of airdropped bodies, from store release until landing. Therefore, DLR has established a multi-fidelity simulation approach which on the one hand allows for computing entire trajectories in real-time ${ }^{14}$. However, as the real-time simulation relies on a low-fidelity aerodynamic model the strong interference effects between the wake of the aircraft and the cargo in the initial stage of the drop are not accounted for. To overcome this drawback, a highfidelity simulation approach was simultaneously developed ${ }^{8}$. It combines state-of-the-art CFD methods with a flight mechanic simulation tool and is specifically applied to investigate the wake interference effects during airdrop. Apart from the numerical approach, accompanying experimental investigations were conducted to provide validation data.

In the present study a validation of the flow topology around several cargo configurations is carried out. Although transient effects may have an influence on the flow ${ }^{15}$, the study was carried out for cargo bodies being rigidly attached to the aircraft. Experimental validation data was obtained in the low speed facility of German-Dutch wind tunnels (DNW-NWB) in Braunschweig, Germany, in 2011 by means of stereoscopic Particle Image Velocimetry (SPIV) ${ }^{16}$. This data basically contains information on the mean and instantaneous velocity field in the near-field of the aircraft and around the cargo bodies. A numerical study employing the DLR TAU code, a Reynolds-averaged Navier-Stokes solver, was conducted. The objective of the study consisted in investigating the influence of four turbulence models in predicting the highly vortical flow and to reveal areas where large discrepancies to the experiment occur. The knowledge gained in this study will be incorporated into DLR's high-fidelity airdrop simulation.

\section{Experimental Flow Field Investigation}

\section{II.A. Wind Tunnel and Models}

Between 2006 and 2012 six wind tunnel campaigns were carried out by DLR in the DNW-NWB low-speed wind tunnel facility in Braunschweig, Germany, to investigate flow field interference effects during airdrop and the trajectories of dropped bodies ${ }^{13}$. The wind tunnel is a closed circuit, atmospheric wind tunnel with open, closed or slotted wall test section. The test section offers a cross section of $3.25 \mathrm{~m} \mathrm{x} 2.8 \mathrm{~m}$. A maximum flow velocity of $90 \mathrm{~m} / \mathrm{s}$ can be set in the closed test section. To allow for an undisturbed flow around the aircraft in the area of interest, the aircraft was supported by a dorsal sting mounted to the "Model Positioning Mechanism" (MPM) of the DNW-NWB ${ }^{17}$. The MPM proved very advantageous during the PIV measurements as the aircraft could easily be moved laterally, requiring only a single PIV calibration of the fixed PIV apparatus. Thus, a very efficient measurement of several planes parallel to the symmetry plane of the aircraft was achieved.

A generic wind tunnel model of a representative military transport aircraft was used. It is manufactured in 1:21 scale and has a wing span of approximately $2 \mathrm{~m}$. The model consists of fuselage, wing, propeller pylons, flap track fairings, a lowered ramp and a T-tail. The propellers themselves are not included. A turbulent boundary layer transition is enforced by zig-zag tape on the nose, the propeller pylons and the leading edges of the wing and the tails. 


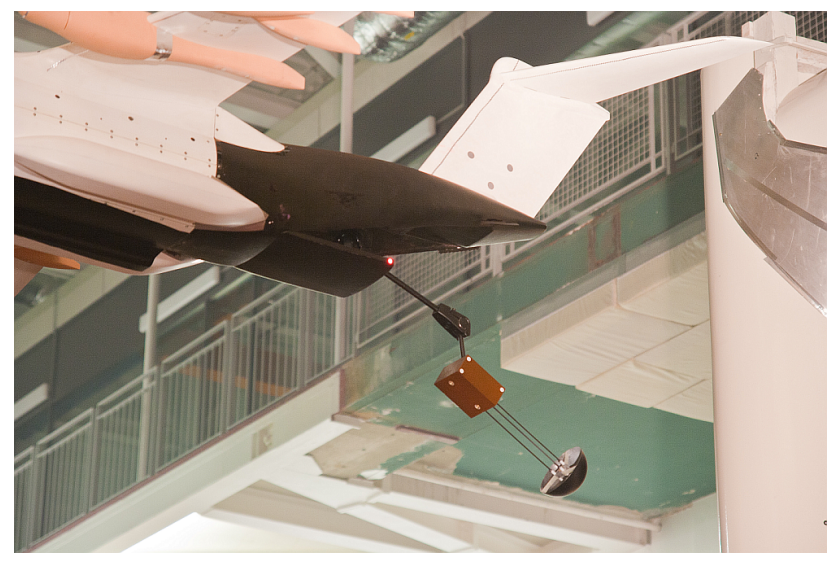

(a) Cargo and parachute in the DNW-NWB wind tunnel

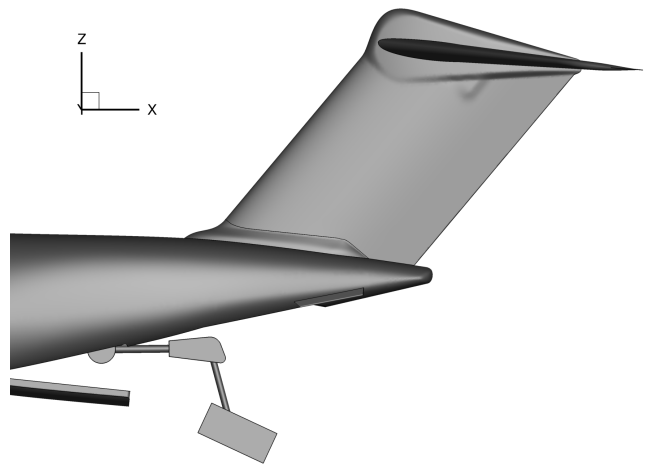

(c) Cargo (Case $C)$

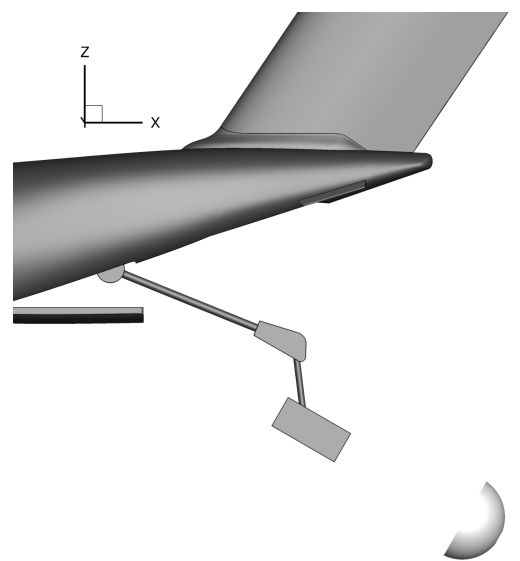

(b) Cargo and parachute (Case B)

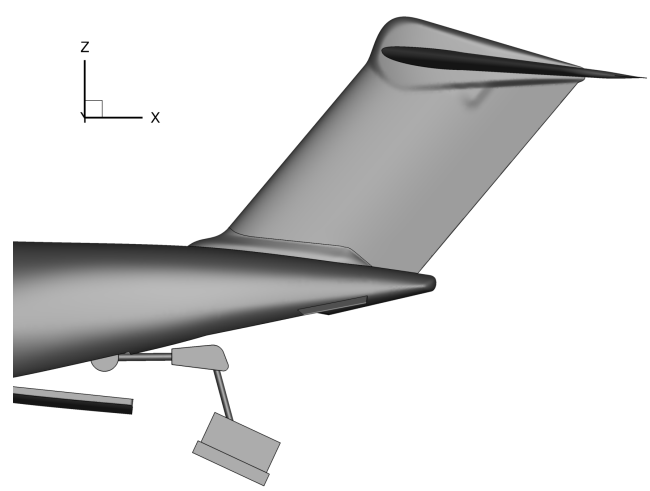

(d) Cargo on support platform (Case D)

Figure 1. Overview on the investigated generic cargo bodies and their position relative to the aircraft. All bodies were symmetrically positioned behind the aircraft.

Figure 1(a) shows the rear part of the aircraft with a rigidly attached cargo configuration.

Three cargo configurations were used, all of which consist of a cuboid main cargo body as seen in Figures 1(b)-1(d) with dimensions of (length x height x width) $110 \mathrm{~mm}$ x $50 \mathrm{~mm} \times 90 \mathrm{~mm}$. The geometric complexity was increased in two ways: first, a simplified model of an inflated drogue parachute was attached to the cargo body as depicted in Figure 1(b). It is modeled by means of a rigid, unslotted hemisphere, containing a vent hole with a diameter of $20 \mathrm{~mm}$ on its symmetry axis. The parachute has an outer diameter of $130 \mathrm{~mm}$ and a constant shell thickness of $1 \mathrm{~mm}$. The inflation process was not taken into account. The hemisphere is rigidly connected to the cargo by means of three carbon-fibre rods with a length of $215 \mathrm{~mm}$ each as illustrated in Figure 1(a). Secondly, a cuboid support platform with dimensions of $120 \mathrm{~mm}$ x $10 \mathrm{~mm}$ $\mathrm{x} 100 \mathrm{~mm}$ was centered below the cargo as seen in Figure 1(d).

To ensure a sufficient number of independent snapshots for a statistically reliable PIV evaluation, it is necessary to statically position the cargo configurations behind the aircraft. A custom-made support mechanism was developed as shown in Figure 1 for that reason. It connects the ceiling of the aircraft cargo bay with the upper face of the cargo body. Two integrated joints allow for multiple positioning possibilities of the cargo in the near-field of the aircraft. The mechanism was designed such to guarantee sufficient stiffness to prevent vibrations of the cargo body. Nevertheless, the existence of the support mechanism leads to unavoidable artificial flow features which are introduced into the near-field wake. In order to minimize these adverse effects, the support mechanism was constructed such that the most voluminous and disturbing components are located in the wake region of the aircraft or within the cargo bay, as depicted in Figures 1(c) and $1(\mathrm{~d})$. In addition, only one linkage bar with reduced diameter is connected to the cargo. 
The contact point of the bar was deliberately chosen as the flow past the upper surface of the cargo is always detached at positive angles of attack. Hence, the additional disturbances of the linkage bar could be minimized as they are induced in an area of already disturbed flow. The experiments were conducted at velocities of $18 \mathrm{~m} / \mathrm{s}, 36 \mathrm{~m} / \mathrm{s}$ and $54 \mathrm{~m} / \mathrm{s}$. While the lowest velocity corresponds to real airdrop scenarios (approx. $82 \mathrm{~m} / \mathrm{s}$, Froude scaling ${ }^{10}$ ), the intermediate velocity allowed for a better quality of the wind tunnel flow. The highest was used for velocity variation purposes only.

\section{II.B. PIV Setup}

Stereoscopic Particle Image Velocimetry (SPIV) was applied to experimentally investigate the flow field. Two coplanar laser light sheets were used to reduce optical blockage effects due to shadows around the cargo bodies. Additionally, the surfaces of the support mechanism, the cargo bodies and the rear fuselage of the aircraft are coated with an acrylic black paint with additives of Rhodamine. Hence, laser light sheet reflections could be minimized. Two cameras were used in stereoscopic arrangement for recording the particle frames. Thus, three-component (3C) mean and instantaneous velocity field information were obtained. Details of the experimental set-up and averaged flow features were published by Roosenboom et al. ${ }^{16}$. For the same experimental campaign a discussion of periodic and instantaneous flow features is published by the same authors $^{18}$. The experimental setup is shown in Figure 2.

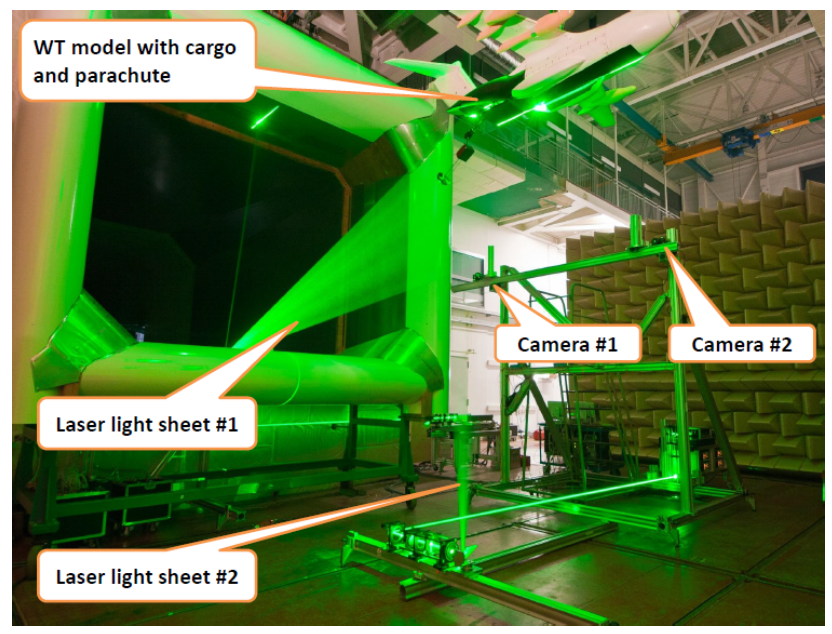

Figure 2. Experimental setup in the open test section of the DNW-NWB wind tunnel.

A combination of various issues resulted in suboptimal PIV conditions. First, the model and the cargo cause shadow regions due to blockage of camera and laser. This was for a large part relieved by using two coplanar lasers. However, at the overlapping region, a gradient of laser light intensities was present. Since the wind tunnel model was traversed in spanwise direction the laser light sheets illuminated several parts of the wind tunnel model and cargo, causing reflections which led to saturation of the particle images. The stereoscopic set-up was in a slightly unfavourable arrangement $\left(90^{\circ}\right.$ and $50^{\circ}$ backward scattering direction). In addition, only limited amounts of seeding material were allowed by the wind tunnel operator because of unknown impact on the acoustic damping material in the recently renovated wind tunnel ${ }^{19,20}$. Therefore, the particle images were post-processed with background image subtraction, high-pass filtering and masking of particular regions ${ }^{16,18}$.

\section{II.C. Summary of Experimental Test Conditions}

A summary of the investigated configurations and onflow conditions is given in Table 2. The experiments were carried out in ambient atmospheric conditions. As the investigation of the configurational impact on the flow topology was of greater importance than the influence of a velocity variation, only the results at $36 \mathrm{~m} / \mathrm{s}$ will be discussed in this paper. This onflow velocity corresponds to a free stream Mach number of $M=0.106$ and a Reynolds number of $R e_{c}=665,000$, based on the reference chord length $c$ of the aircraft. 
Table 2. Experimental Test Conditions (bold conditions are discussed within this paper)

\begin{tabular}{llcccc}
\hline \hline Case & Configuration & \multicolumn{2}{c}{ Abs. angle of attack } & $U_{\infty}$ & PIV plane location $(y)$ \\
& & Aircraft $\left[^{\circ}\right]$ & Cargo $\left[^{\circ}\right]$ & {$[\mathrm{m} / \mathrm{s}]$} & {$[\mathrm{mm}]$} \\
\hline A & Aircraft only & 0 & - & $18 ; 36$ & $10,35,60,90$ \\
B & Aircraft, cargo, parachute & 0 & 30 & $18 ; \mathbf{3 6}$ & $\mathbf{1 0}, 35,60,90$ \\
C & Aircraft, cargo & 6 & 25 & $18 ; \mathbf{3 6} ; 54$ & $\mathbf{0}, 20,30,70$ \\
$\mathbf{D}$ & Aircraft, cargo, platform & 6 & 25 & $\mathbf{3 6} ; 54$ & $\mathbf{0}, 30,70$ \\
\hline \hline
\end{tabular}

\section{Numerical Flow Field Investigation}

\section{III.A. Geometry and Grid Generation}

The grids were generated with the hybrid grid generator Centaur, a commercial software package developed by CentaurSoft ${ }^{21}$. Half-model meshes were generated such to to yield a non-dimensional first wall spacing of $y^{+}=1$ for the respective bodies, according to the local Reynolds number. The latter was determined based on the reference chord length $c$ of the aircraft, yielding $R e_{c}=665,000$ for the aircraft. Based on the span $b$ of the aircraft the computational domain is closed by a box-type farfield boundary, being $50 \cdot b$ away from the aircraft origin in all directions. As for the cargo bodies, the local Reynolds number was determined in using the height $h$ of the front face of the cuboid cargo body, leading to a local Reynolds number of $R e_{h}=123,000$. The tetrahedral mesh within the cargo bay, in the near-field region behind the aircraft and around the cargo bodies was refined with uniform tetrahedra, having an edge length of approx. 1.5\% of $c$. The resulting grid features a very high resolution of the wake region. By using the same sources for all configurations, highly identical meshes were obtained. The resulting half-model meshes were mirrored to obtain the full-model meshes. The final meshes contain around $60,000,000$ nodes. Figure 3 gives an impression on the mesh resolution in the wake of the aircraft for Case B. Moreover, the location of the PIV planes discussed in this paper are shown.

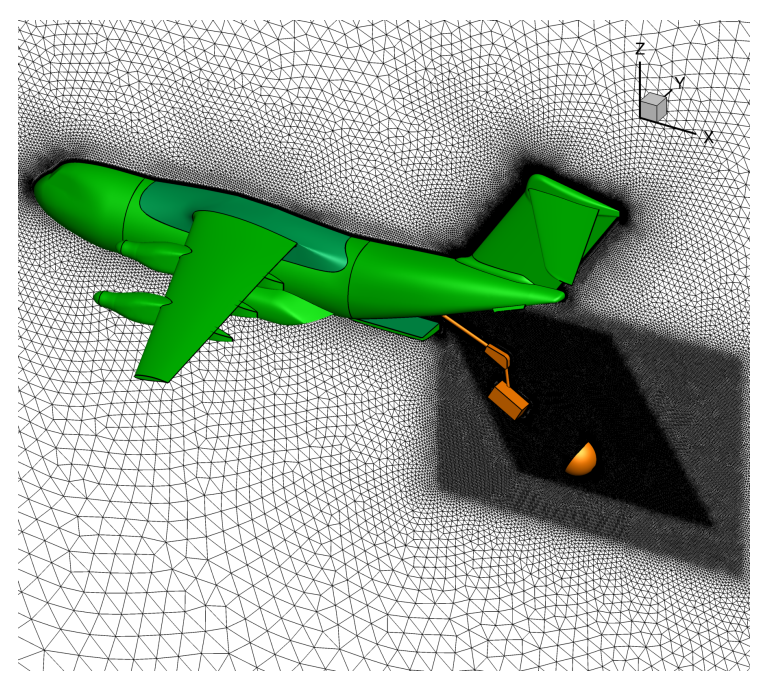

(a) Grid overview

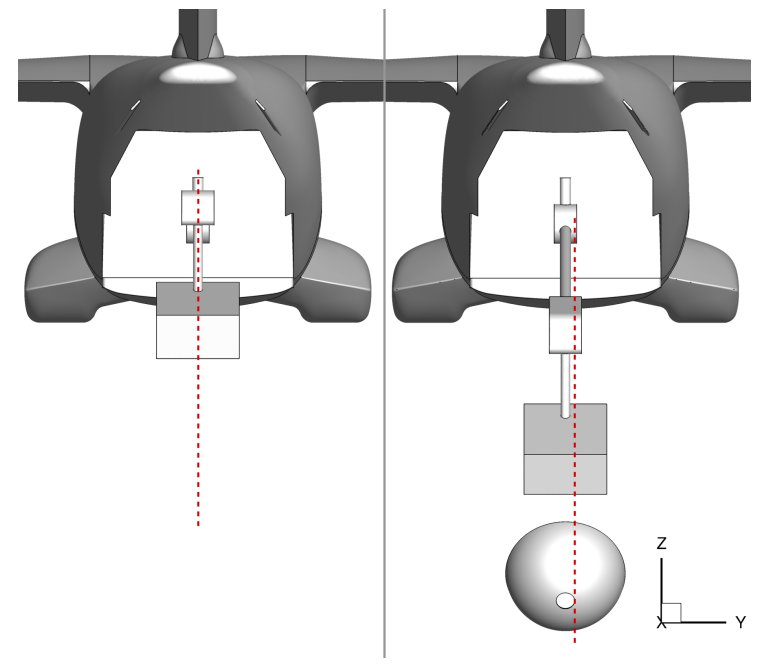

(b) Location of PIV planes for Cases $C$ and $D$ (left) and Case $B$ (right)

Figure 3. Exemplary overview of the grid resolution in the wake of Case B and illustration of the PIV planes being discussed in this paper.

\section{III.A.1. Differences to Experimental Setup}

Two main differences occur compared to experiment: the first is found in the CAD model of the support mechanism that connects the cargo bay ceiling with the cargo body. As it is located completely within the cargo bay, the support mechanism in CFD is not connected to the ceiling of the cargo bay to ease the mesh generation process and to save grid points. Secondly, the three carbon-fibre rods, connecting the parachute 
model with the cuboid cargo as shown in Figure 1(a), have been neglected as well to keep the overall mesh size within limits. Consequently, the support frame within the experimental parachute model, to which the three rods are connected to, has also been omitted in CFD. Thus, the experimental parachute shows more pronounced blockage effects than its CFD counterpart and the carbon-fibre rods induce additional flow features which have been recorded by PIV, but are missing in the computed data. The final CFD setup for this body is shown in Figure 1(b).

\section{III.B. The DLR TAU Code}

All computations were performed with the DLR TAU code, a Reynolds-averaged Navier-Stokes (RANS) solver which is under continuous development by $\mathrm{DLR}^{22-24}$. The main developments of the TAU code originated from the German CFD project MEGAFLOW ${ }^{25,26}$, consolidating contributions from DLR, the aeronautical industry and universities. TAU is an unstructured, edge-based solver in cell-vertex or cell-centered formulation. A dual-grid approach is used to allow for flow computations on grids being independent of the initial grid cell types. TAU solves the compressible, three-dimensional, time-accurate RANS equations. The spatial discretization, based on an upwind scheme or a central scheme with artificial scalar or matrix dissipation, is based on a finite volume formulation. The temporal discretization may either be carried out with an explicit Runge-Kutta or an implicit Lower-Upper Symmetric Gauss-Seidel (LU-SGS) scheme. Several convergence acceleration techniques like residual smoothing or multigrid may be applied. A vast number of turbulence models has been implemented, ranging from algebraic turbulence models up to differential Reynolds stress models. Today, TAU is routinely used in the European aeronautical industry for external aerodynamics.

Steady RANS computations were carried out for all cases listed in Table 2, relying on the one-equation turbulence model by Spalart and Allmaras ${ }^{27}$ (SA) and the two-equation Menter $k \omega$-SST ${ }^{28}$ model (SST). The most complex case from an aerodynamic point of view, Case B, was additionally computed with more advanced turbulence models which have shown promising results in capturing challenging flow phenomena in the past. These are on the one hand the SA model with rotational and curvature correction ${ }^{29}$ (SARC) which for instance performed very well in capturing vortex dominated flows ${ }^{30}$. On the other, the SSG/LRR- $\omega$ differential Reynolds stress model ${ }^{31}$ (RSM) is used. Of all four models the RSM provides the most accurate physical modelling of turbulence as it accounts for its anisotropic nature by directly computing the different components of the Reynolds stress tensor. Thus, it is specifically suited for detached or vortical flows. Its implementation in TAU revealed promising results for transport aircraft configurations as presented in several DLR publications ${ }^{32-34}$. Besides the SARC, it was therefore applied as well to compare the influence and the potential advantages of the two more advanced models over the standard SA and SST models. All computations rely on the Jameson-type central scheme ${ }^{35}$ with artificial matrix dissipation for the spatial discretization. The implicit LU-SGS scheme was applied for the temporal discretization. Furthermore, unsteady RANS computations were carried out to assess its impact in resolving the flow time-accurately as opposed to the steady approach. The unsteady computations were limited to Case B using the SARC model only to save computational time.

\section{III.C. Convergence Behaviour}

In general, a good and robust convergence behaviour was achieved for all configurations. The density residual decays by $4-7$ orders of magnitude, with the only exception being the RSM. Using the latter a decay of only two orders of magnitude was achieved. Although massive flow separation and vortices are present in the flow field, the force and moment coefficients converge towards a steady value, though with more evident oscillations in case of the configurations with parachute. Therefore, both mean and root mean square (RMS) values have been computed over the last $10 \%$ of the computed iterations to compare the convergence behaviour of the different models.

The oscillations were found to be negligible for the configurations without parachute, with maximum RMS values of lift and drag coefficients being below $0.1 \%$ of the resulting mean values, corresponding to $\Delta C_{L} \leq 4 \cdot 10^{-5}$ and $\Delta C_{D} \leq 5 \cdot 10^{-5}$. The configuration with parachute, in contrast, yields more pronounced oscillations in lift, drag and density residual. The highest oscillations in the lift coefficient are observed for the SST model, showing RMS values of up to $\Delta C_{L} \leq 8 \cdot 10^{-4}$, corresponding to $5.5 \%$ of the mean value. For the drag coefficient the RSM shows the highest oscillations with variations of up to $\Delta C_{D} \leq 9 \cdot 10^{-4}$, i. e. $1 \%$ of the mean value. 


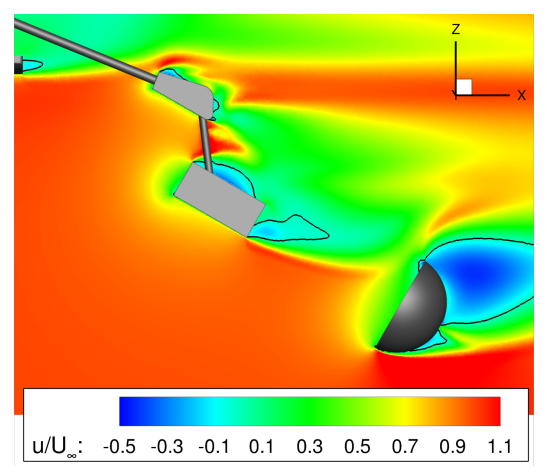

(a) SA

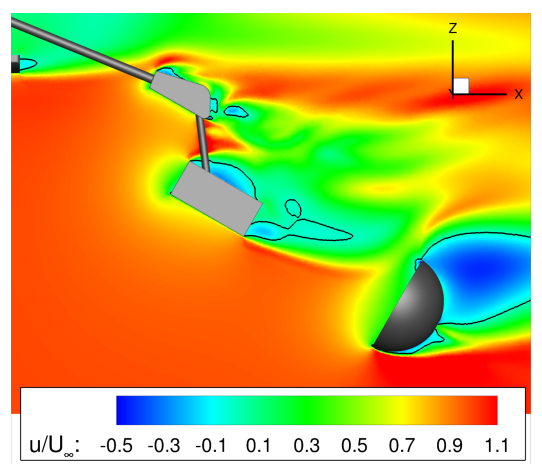

(d) SARC

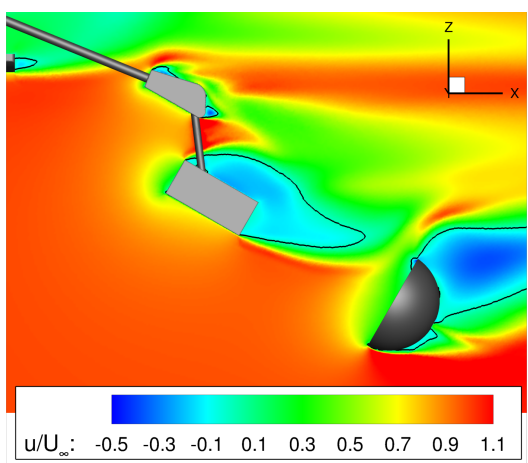

(b) $\mathrm{SST}$

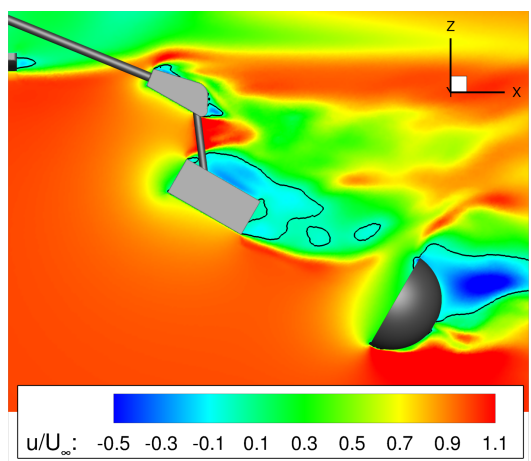

(e) RSM

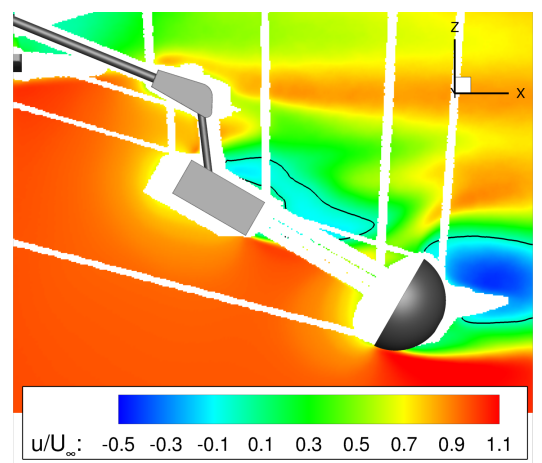

(c) PIV

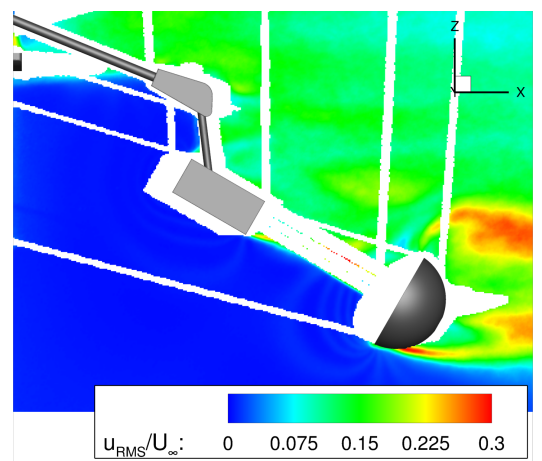

(f) PIV RMS

Figure 4. Absolute $x$-velocity component $u$ for CFD and 3C-PIV at $y=10 \mathrm{~mm}$ off the symmetry plane for Case B, normalized with the onflow velocity of $U_{\infty}=36 \mathrm{~m} / \mathrm{s}$. In addition, the PIV RMS values are shown. The black solid line represents the iso-line where $u=0$.

\section{Validation of the Numerical Simulation}

The validation is based on comparing the distribution of the mean velocity vector components. For the sake of clarity, only a few selected of the cases mentioned in Table 2 will be discussed in this paper. Cases indicating high RMS velocities have been preferred. Therefore, Case $A$ is omitted here as the differences among the turbulence models and between CFD and PIV are negligible. Case B, in contrast, is the most challenging from an aerodynamic point of view. It will therefore be discussed in greater detail to examine the performance and limitations of steady and unsteady RANS methods. Note on the masked regions in the PIV images: the stereoscopic PIV camera arrangement and the lateral displacement of the wind tunnel model with respect to the cameras leads to double masked regions in the mapped field of view, caused by different lines of sight of the left and the right camera.

\section{IV.A. Steady Results for Case B: Aircraft, Cargo and Parachute}

\section{IV.A.1. Absolute mean velocity distribution}

The normalized absolute velocity components were evaluated to get a first impression on the qualitative agreement between CFD and PIV. Figure 4 shows the mean velocity distribution in $x$-direction at $y=10 \mathrm{~mm}$ off the symmetry plane. After evaluating the PIV data, Roosenboom et al. identified several regions of characteristic flow topologies for this configuration ${ }^{16}$ : (i) the wake of the joint of the support mechanism, (ii) the wake emerging from the upper surface of the cargo body, (iii) the local flow displacement ahead of the parachute and (iv) the wake of the parachute canopy. These four regions are clearly captured in CFD, as shown in Figs. 4(a) - 4(e). In addition, the PIV RMS values of the $x$-velocity depicted in Fig. 4(f) reveal oscillations in the entire wake of the bodies at a magnitude of $5 \%-20 \%$ of the respective mean values. The wake of the parachute shows an even higher magnitude, up to $25 \%-30 \%$, indicating that a pronounced flow unsteadiness is present there.

In this context it should be underlined that, unlike PIV, the chosen steady RANS approach is limited in 


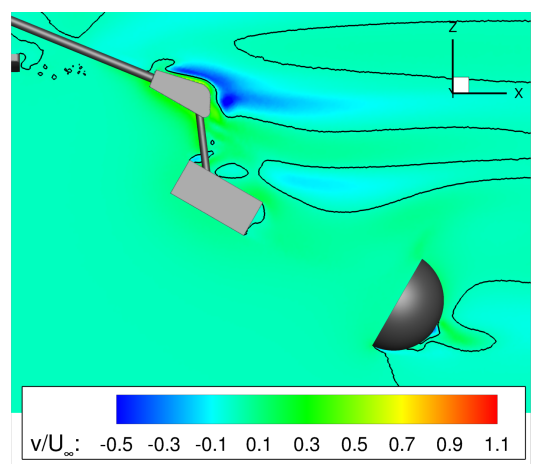

(a) SA

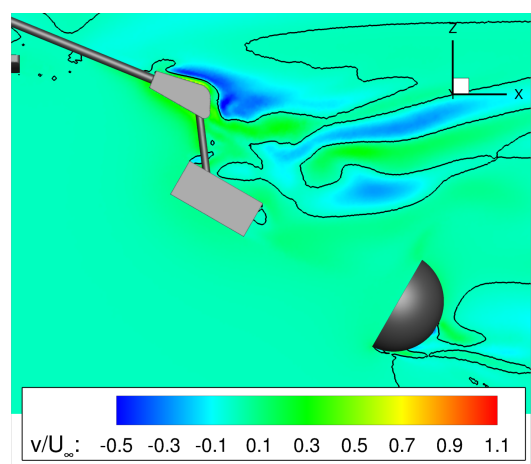

(d) SARC

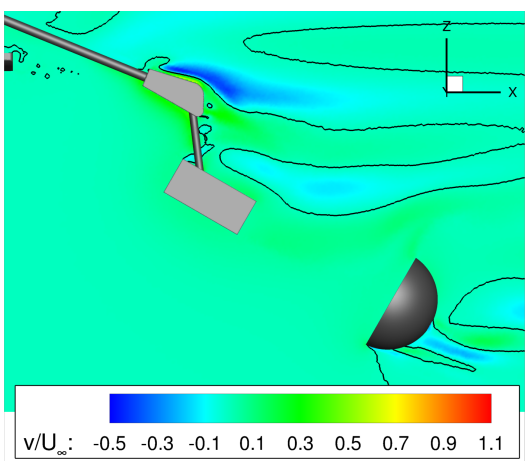

(b) $\mathrm{SST}$

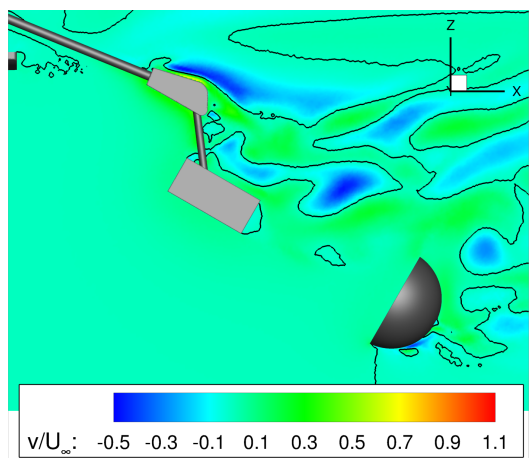

(e) RSM

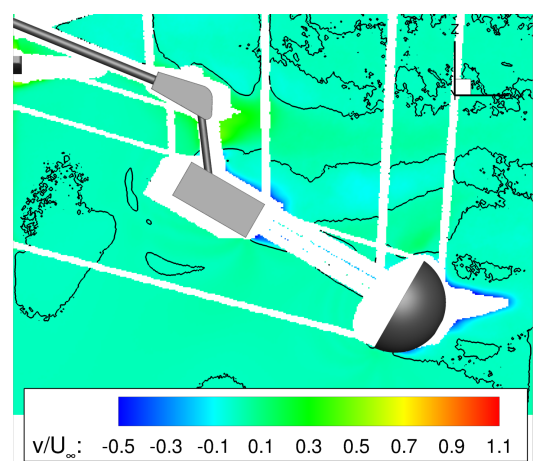

(c) PIV

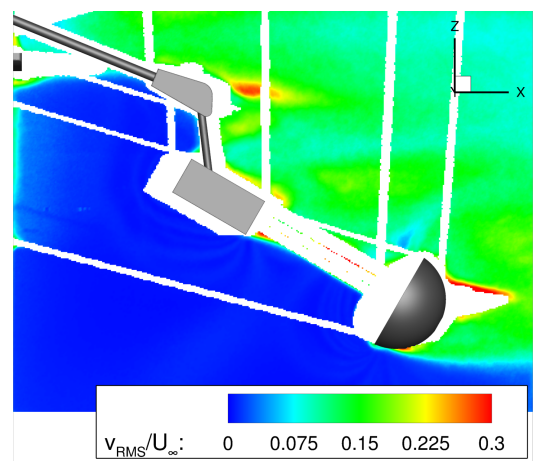

(f) PIV RMS

Figure 5. Absolute $y$-velocity component $v$ for CFD and 3C-PIV at $y=10 \mathrm{~mm}$ off the symmetry plane for Case B, normalized with the onflow velocity of $U_{\infty}=36 \mathrm{~m} / \mathrm{s}$. In addition, the PIV RMS values are shown. The black solid line represents the iso-line where $v=0$.

capturing these unsteady flow effects. The reader shall therefore be reminded again on the objective of this study, which in a first step consists in identifying the capabilities and limitations of steady RANS methods for such configurations. Of course, the question arises in how far the results could be improved by applying more appropriate methods to resolve the unsteady effects. This question will be answered further below in section IV.B.

At first glance, the qualitative agreement between CFD and PIV is very good, the aforementioned regions are all captured well by the simulations. A closer look into the wake regions of the bodies reveals slightly higher CFD flow velocities. This becomes most significantly evident in the wake of the lower linkage bar. Regarding the flow past the parachute, the local flow displacement just ahead of the parachute due to the adverse pressure gradient introduced by it is also well predicted by CFD. Nevertheless, differences occur behind the parachute, as seen near the vent hole on the parachute symmetry axis. The simulations resolve a clearly visible jet through this vent hole, while the latter is not present in the PIV results.

As will be shown in section IV.A.2 this can be traced back to geometric differences between the numerical and the wind tunnel setup. The investigation of the size and the location of the separated flow regions, indicated by the black lines representing the location of $u=0$, are also well predicted by the simulation when it comes to the parachute. The RSM model predicts the smallest size of that region. Regarding the separation on the cargo body, the SST model shows an excellent agreement with the experimental data. All other turbulence models predict much smaller regions of flow separation.

Figure 5 shows a comparison of the $y$-velocities. It becomes evident that the general velocity magnitude in this direction is much lower than in $x$ - or $z$-directions. Although an out-of-symmetry plane is considered only minor cross flow features, e. g. due to vortex shedding, are present in the PIV results shown in Fig. 5(c). The CFD data largely complies very well with PIV, apart from a region above the joint of the support mechanism. There all turbulence models predict a distinct band of negative $y$-velocity, indicating that the local flow is directed towards the symmetry plane. A closer look at Figs. 5(a) - 5(e) unveils that this region appears just beyond the prismatic boundary layer mesh. Within the boundary layer mesh the agreement between CFD and PIV is much better, indicated by the green sublayer. Thus, the mesh resolution in this region 


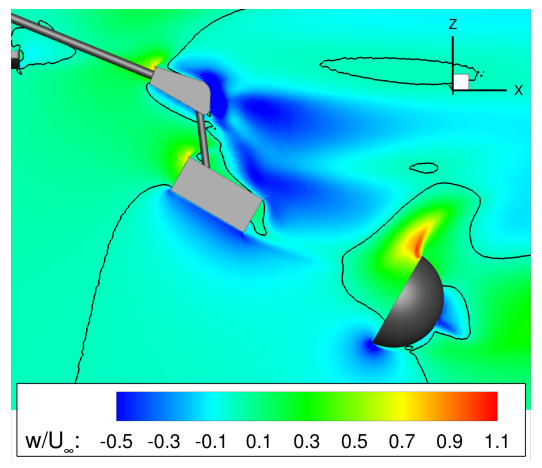

(a) SA

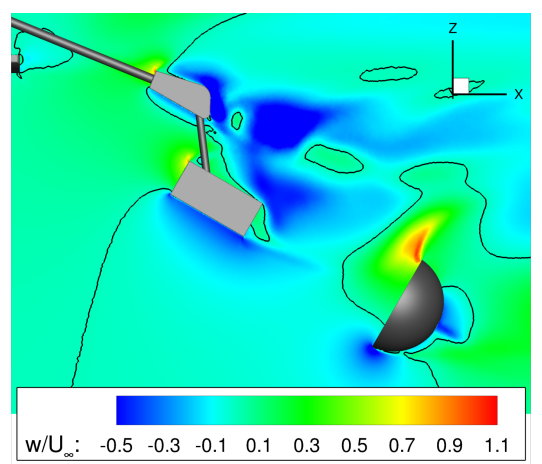

(d) SARC

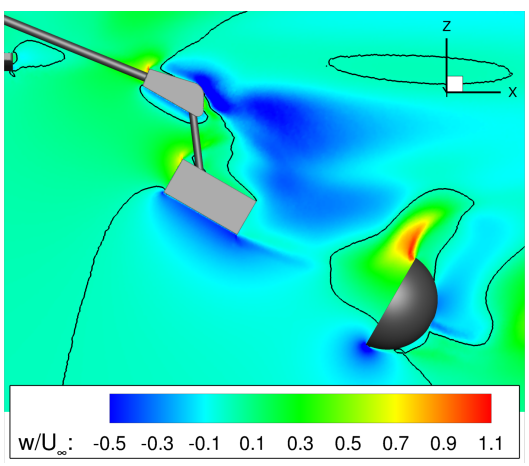

(b) $\mathrm{SST}$

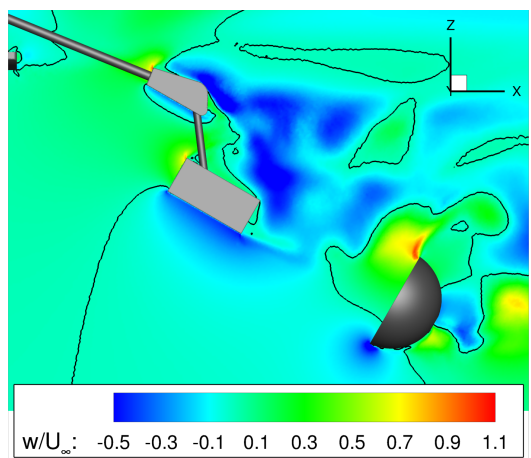

(e) RSM

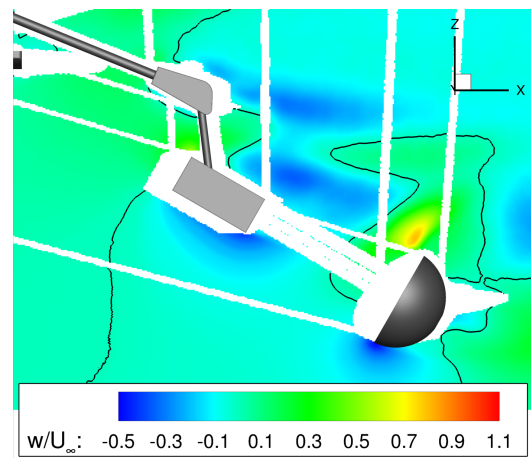

(c) PIV

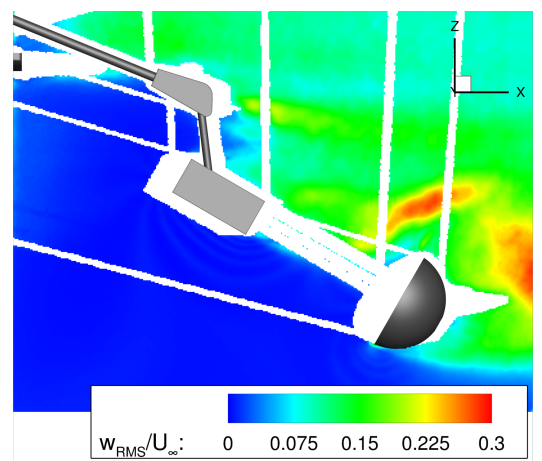

(f) PIV RMS

Figure 6. Absolute $z$-velocity component $w$ for CFD and 3C-PIV at $y=10 \mathrm{~mm}$ off the symmetry plane for Case B, normalized with the onflow velocity of $U_{\infty}=36 \mathrm{~m} / \mathrm{s}$. In addition, the PIV RMS values are shown. The black solid line represents the iso-line where $w=0$.

obviously needs to be improved to properly resolve the emanating flow features from the front side of the joint. Regarding the RMS values in Fig. 5(f), oscillations are fewer than before but still appear, especially behind the joint and behind the parachute. The general background intensity is nearly on the same level as seen before in Fig. 4(f).

Fig. 6 reveals the respective $z$-velocities and the associated PIV RMS data. The overall magnitude of this velocity component has increased again. In general, Figs. 6(a) and 6(b) reveal a good qualitative agreement between the SA and SST results and the PIV result in Fig. 6(c). The region of downstream flow in the wake of the support mechanism and the cargo body is similarly developed in size, though slightly overpredicted in magnitude by CFD, i. e. CFD shows a stronger downward component. As seen in Figs. 6(d) and 6(e) the wake prediction by the SARC and RSM models in that region is speckled, as opposed to a more continuous region in case of the SA and SST models. A marginal overprediction of the velocity magnitude also takes place near the upper edge of the canopy where the turbulence models show a very high component in upward direction. The RMS data in Fig. 6(f) reveals a significant flow unsteadiness which predominantly appears in the wake region of the parachute and above the parachute where the flow is displaced in upward direction.

\section{IV.A.2. Differences in the distribution of the velocity magnitude}

The differences in the velocity magnitude are displayed in Fig. 7 to have additional information to assess the performance and accuracy of the different turbulence models in predicting the correct flow field. These differences were computed according to Eq. (1), i. e. differences of $\Delta V>0$ indicate regions where PIV predicts a higher velocity magnitude than CFD. Consequently, in regions where $\Delta V<0$ PIV predicts a lower velocity magnitude.

$$
\Delta V=V_{P I V}-V_{C F D}
$$

A look at Fig. 7 reveals three regions in which strong velocity deviations appear: (i) the interacting wakes of the lower support mechanism and the upper side of the cargo, (ii) the shear layer emerging from the lower 


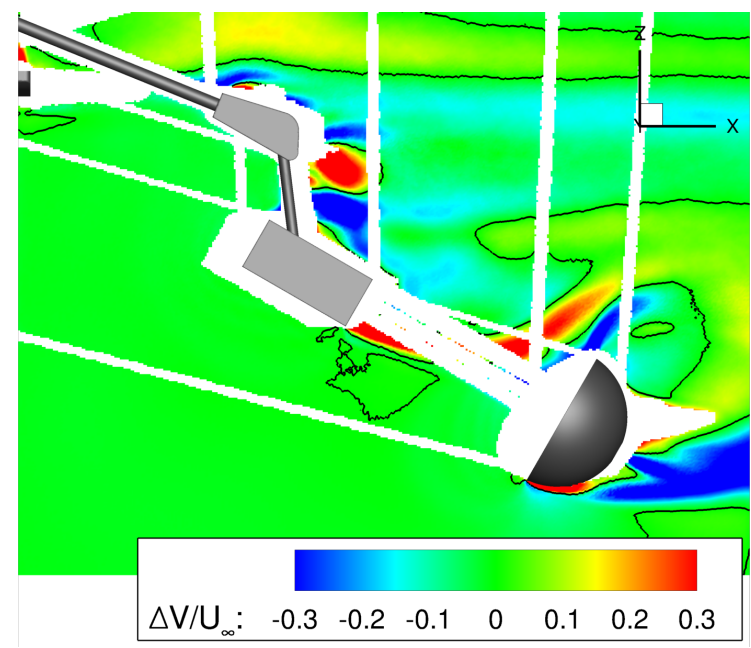

(a) PIV-SA

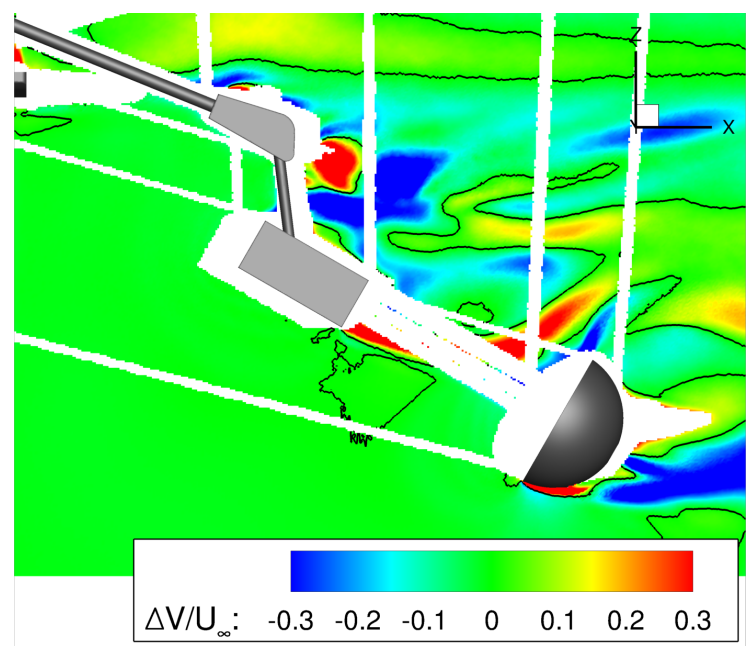

(c) PIV-SARC

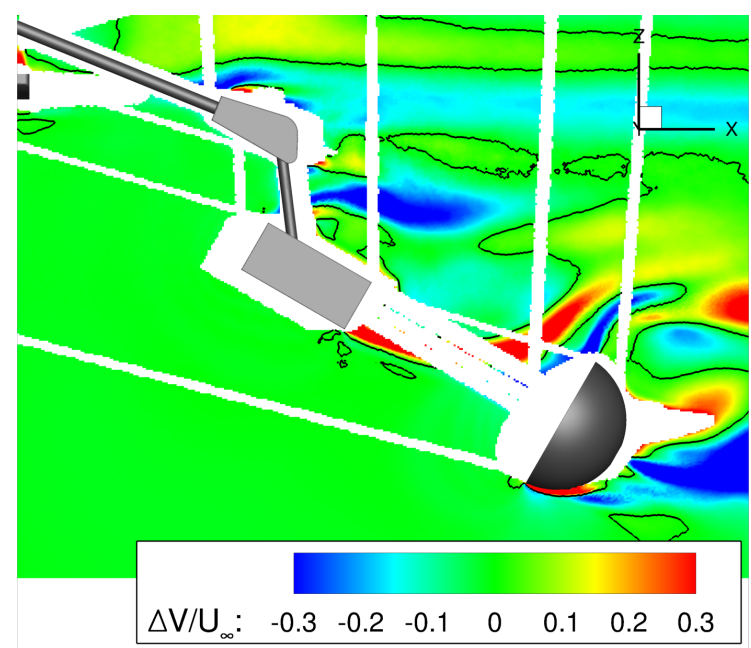

(b) PIV-SST

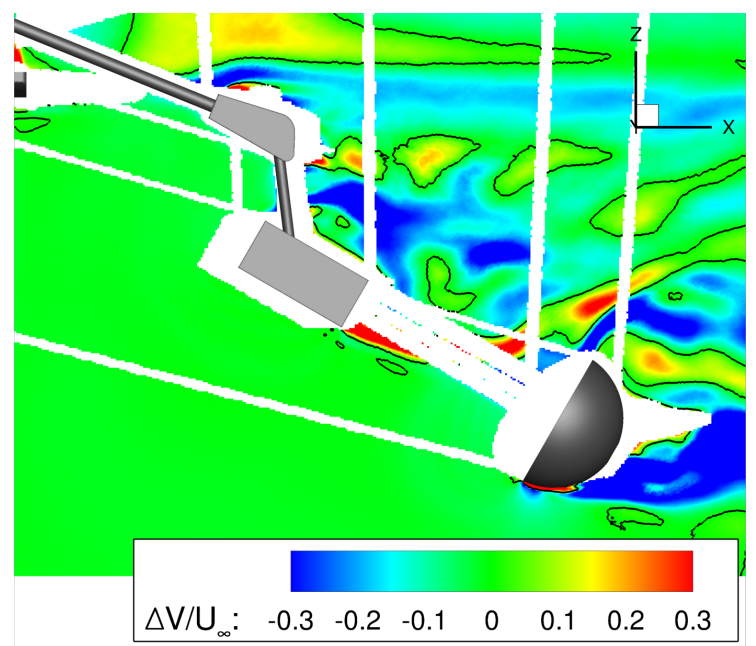

(d) PIV-RSM

Figure 7. Differences of the velocity magnitude $V$ at $y=10 \mathrm{~mm}$ off the symmetry plane for Case $B$, normalized with the onflow velocity of $U_{\infty}=36 \mathrm{~m} / \mathrm{s}$. The black line indicates the location with $\Delta V=0$.

side of the cargo and (iii) the flow past and the wake of the parachute. In those regions, deviations of around $30 \%$ of the onflow velocity can be identified.

In the first region it can be observed that the simulation produces a higher velocity magnitude in the immediate wake of the lower linkage bar, as shown by the deep blue regions above the cargo in Figs. 7 (a) $-7(\mathrm{~d})$. As discussed in the last section CFD predicts a local absolute $x$-velocity in this region being higher than the onflow velocity. Consequently, a strong acceleration of the flow takes place which is due to the local arrangement of the geometry. Just upstream of the lower linkage bar a contraction of the effective height, which the flow must pass, is formed by the lower side of the joint and the upper side of the cargo. Hence, the local flow faces conditions typically encountered in nozzle flows. The throat of the nozzle, i. e. the location with the smallest effective height, is located just upstream of the lower linkage bar. As a consequence an acceleration takes place in that region. The particular reason for the higher velocities in CFD is twofold: on the one hand the size and shape of the region of separated flow on the upper side of the cargo further decreases the effective throat height, leading to a more pronounced acceleration of the flow. On the other the mesh resolution in that zone might still be too coarse to more accurately capture the interacting flow features. The results of SA and SST models are closest to the experimental data.

Focusing on the second region, i. e. the shear layer emanating from the lower side of the cargo, further significant deviations become evident. As visualized by the red band behind the cargo the PIV velocities exceed the ones predicted by CFD. As the PIV RMS values for the three velocity components remain 
at low magnitude in that area as illustrated in Figs. 4(f), 5(f) and 6(f), these differences are probably not associated to an unsteady flow behaviour. Instead, a glance at the velocity components in $x$ - and $z$ directions in Figs. 4 and 6 discloses that both CFD and PIV predict a similar magnitude of the velocity components there. What is different, though, is the location and the direction of the departing shear layer. In CFD a nearly tangentially departing shear layer from the lower side of the cargo can be observed in Fig. 4. The corresponding PIV velocity distribution, in contrast, reveals a kink near the lower aft edge of the cargo which leads to a deflection of the shear layer into upward direction. Thus, PIV reveals yet higher flow velocities in regions in the wake of the cargo where the CFD data already predicts reduced flow velocities. As a consequence, this difference in the spatial position of the shear layer explains the significant deviations in the velocity magnitude shown in Fig. 7. The reason for these deviations probably consists in a small misalignment between the experimentally investigated and the numerically simulated geometries. Nevertheless, applying a local grid adaption certainly would narrow the gap between CFD and PIV in that region. Further downstream the shear layer is deflected into upward direction due to the adverse pressure gradient induced by the canopy. In that region, just above the canopy, high $w$-RMS values can be observed as shown in Fig. 6(f). Thus, additional deviations between PIV and the steady RANS computations are introduced in that region due to a locally unsteady flow behaviour. In general, it can be said that the RSM model performs best at capturing the correct absolute flow velocity in the region where high RMS values are observed.

In the third region, i. e. around and behind the parachute, the level of geometric details plays an important role as a cause for some of the differences observed there. As mentioned earlier, the CFRP rods and the attached structure to support the parachute within the canopy are not modeled in CFD. Thus, the blockage effects for the flow inside of the canopy are less pronounced, resulting in higher CFD jet velocities through the vent hole and, consequently, discrepancies compared to PIV. Apart from the jet the entire flow past the upper and lower edges of the canopy shows moderate to strong quantitative deviations. All turbulence models show a higher velocity magnitude around the upper edge of the canopy, indicated by the blue regions. The SARC is closest to experiment. However, taking into consideration the very high RMS values in all spatial directions as shown in Figs. 4(f), 5(f) and 6(f) it becomes obvious that significant velocity fluctuations are present around the canopy due to vortex shedding and flow separation. The highest RMS values are in the range of $20 \%-30 \%$ of the onflow velocity, i. e. approx. $7 \mathrm{~m} / \mathrm{s}$ to $11 \mathrm{~m} / \mathrm{s}$. These highly unsteady effects cannot be accurately captured by the steady simulation approach.

To summarize the performance and the limitations of the applied steady approach for this challenging configuration, it can be stated that the simulations seem to be capable of predicting similar absolute velocities as seen in Figs. $4-6$. However, no turbulence model was capable of reproducing the correct size and shape of the separated flow regions behind the cargo and the parachute. While the SST model showed an excellent agreement around the cargo, Fig. 4(b), it failed in more accurately computing the reversed flow region behind the canopy. In contrast, the SA and SARC models performed very well in that latter region, as depicted in Figs. 4(a) and 4(d), nonetheless both show a poor performance in predicting the correct shape and extension of the wake around the cargo body.

\section{IV.B. Unsteady Results for Case B: Aircraft, Cargo and Parachute}

An exemplary unsteady RANS simulation was performed for Case $B$ with the SARC model to examine if the solution accuracy could be improved by accounting for time-accurate flow conditions. The numerical settings remained unchanged, the unsteady simulation was based on the converged steady SARC solution. The unsteady time step size $\Delta t$ was derived such to be able to resolve the vortex shedding around the lower linkage bar, which features a circular cross section with a diameter of $d=10 \mathrm{~mm}$. Assuming that this cylindrical part is immersed in the undisturbed flow field far below the aircraft a local Reynolds number can be computed with the onflow velocity of $U_{\infty}=36 \mathrm{~m} / \mathrm{s}$. Considering the ambient atmospheric conditions a value of $R e_{\text {local }}=21,000$ is obtained. Based on the relation between Reynolds number and Strouhal number for the flow past a cylinder a non-dimensional vortex shedding frequency of $S r=0.2$ was assumed. With the diameter of the lower linkage bar as characteristic length a dimensional vortex shedding frequency of $f_{\text {shedding }}=720 \mathrm{~Hz}$ is obtained. To properly resolve the relevant flow features the Nyquist criterion must be fulfilled, stating that the resolved frequency needs to be at least twice as high as the shedding frequency. In other words, the Nyquist criterion is fulfilled as long as $\Delta t$ in CFD does not exceed $0.7 \mathrm{~ms}$ in our case. The uRANS simulation was conducted with a time step size of $\Delta t=0.5 \mathrm{~ms}$, corresponding to a sampling frequency of $f=2000 \mathrm{~Hz}$ or $f=2.8 \cdot f_{\text {shedding. }}$. In total, nearly 0.5 s physical time was computed with these 


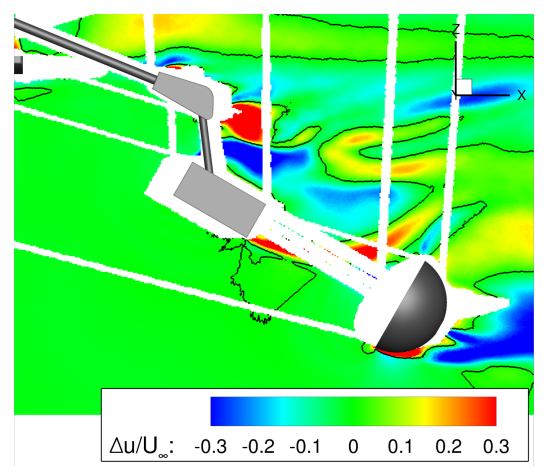

(a) $\operatorname{PIV-SARC}(\Delta u$, RANS)

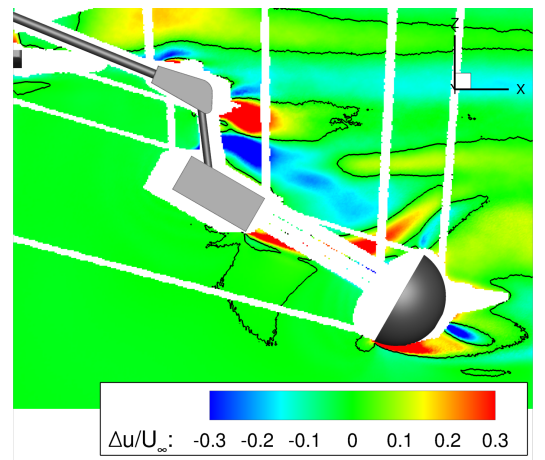

(d) PIV-SARC $(\Delta u$, uRANS)

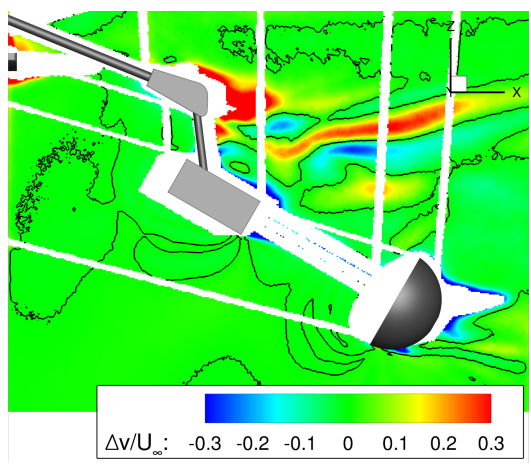

(b) PIV-SARC $(\Delta v$, RANS)

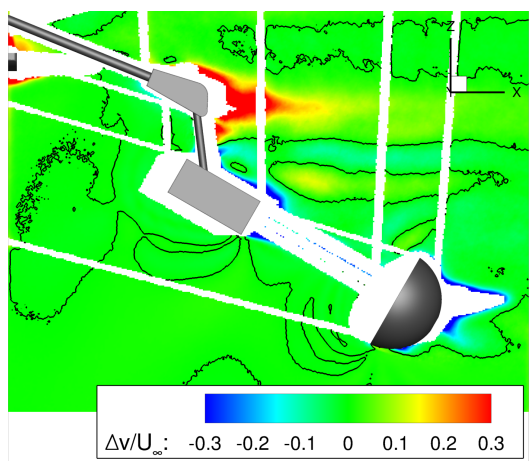

(e) PIV-SARC ( $\Delta v$, uRANS)

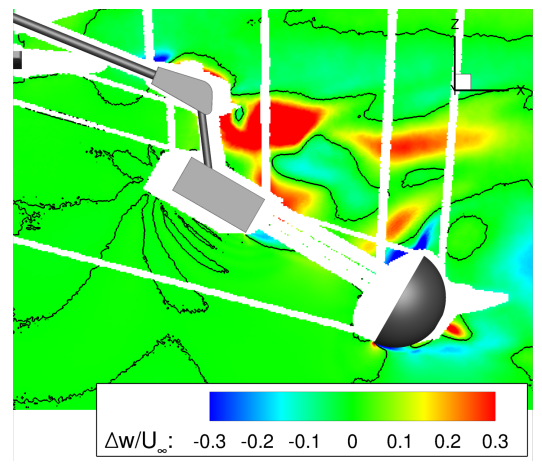

(c) PIV-SARC $(\Delta w$, RANS)

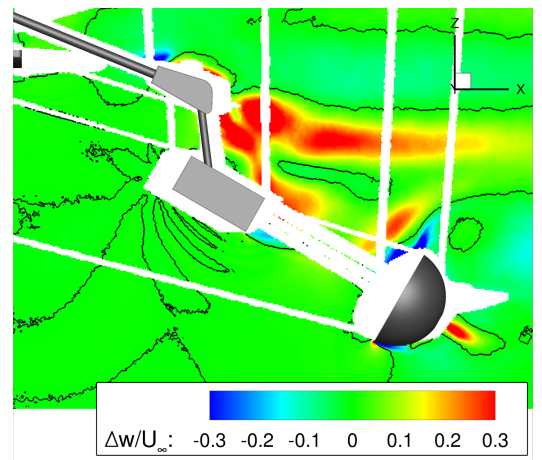

(f) PIV-SARC $(\Delta w$, uRANS)

Figure 8. Influence of the simulation approach on the accuracy of the solution. Shown are the differences between the mean PIV velocity components and the mean RANS (top) or uRANS (bottom) results at $y=10 \mathrm{~mm}$ off the symmetry plane for Case $\boldsymbol{B}$. The data is normalized with the onflow velocity of $U_{\infty}=36 \mathrm{~m} / \mathrm{s}$.

settings.

Figure 8 depicts the differences between the steady and unsteady SARC velocity components compared to PIV. In $x$-direction, Figs. $8(\mathrm{a})$ and $8(\mathrm{~d})$, it becomes evident that the differences in the immediate wake of the joint of the support mechanism and in the flow past the upper front edge of the cargo nearly remain unaffected. Thus, the time-accurate simulation does not offer any benefit in achieving a better solution quality in that zone. Obviously, there must be another reason for the apparent differences, which probably can be attributed to the mesh resolution in that area. The same observations hold for the shear layer from the lower cargo side. Nonetheless, it can also be observed that the general deviations in the wake of support mechanism, cargo and parachute are much less pronounced in the uRANS simulation. The flow field appears more homogeneous than before. The most significant improvement can be recognized in the wake of the parachute where the solution accuracy could be considerably improved.

In $y$-direction, Figs. 8(b) and 8(e), the general flow field in the wake of the bodies appears more uniform in case of the uRANS simulation as discovered before. The strong deviations behind the lower linkage bar have nearly vanished. No changes occur behind the canopy though. The overall agreement to the PIV results is better.

Figures $8(\mathrm{c})$ and $8(\mathrm{f})$ reveal the results in $z$-direction. Slightly larger deviations between uRANS and PIV can be noticed. This holds especially for the wake of the joint where uRANS yields one continuous region of deviations, whereas the RANS approach yielded two separated areas of same magnitude. The reason consists in the fact that, once again, the flow field is better resolved using uRANS, leading to a more uniform flow field and, hence, a more continuous region with deviations. The differences in the wake of the canopy have nearly vanished in the uRANS simulation, apart from the jet through the vent hole. As stated earlier, the latter is due to geometric differences between CFD and PIV.

To summarize, the accuracy of the numerical simulation could largely been enhanced by applying timeaccurate methods. Nevertheless, distinct regions with large deviations remain. Therefore, one can assume that in order to further narrow the gap between CFD and PIV the mesh resolution needs to be adjusted. 


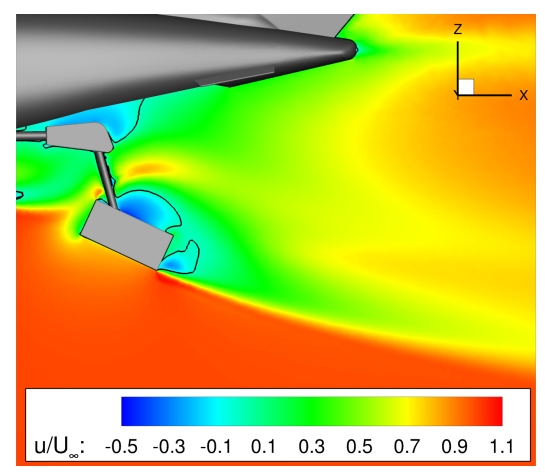

(a) SA

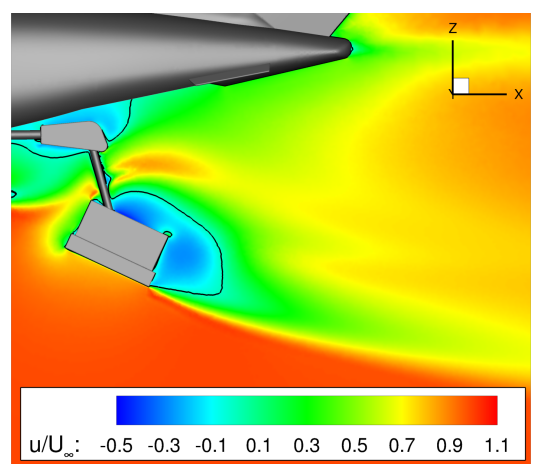

(d) SA

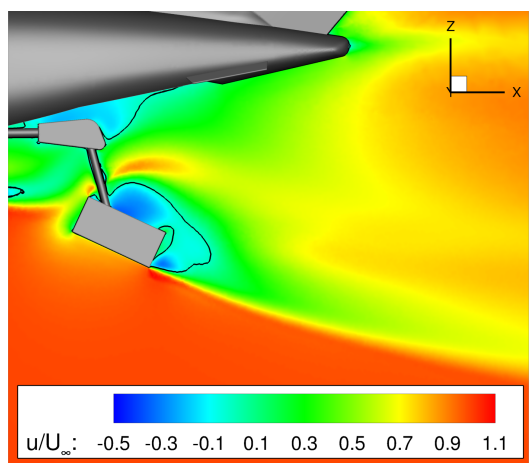

(b) SST

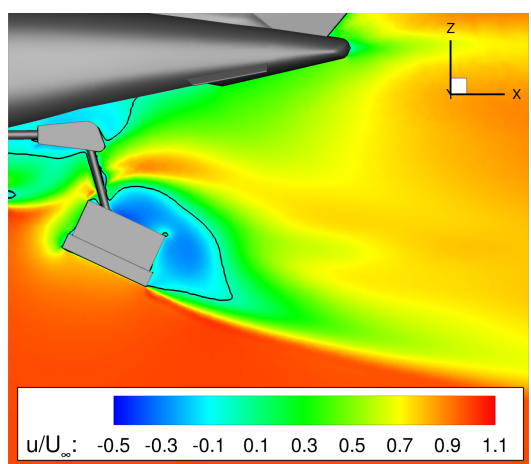

(e) SST

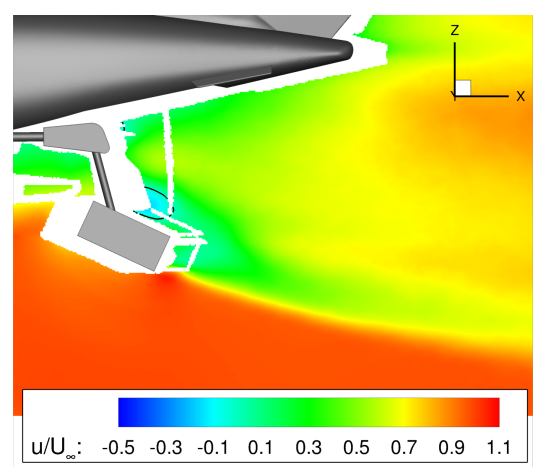

(c) PIV

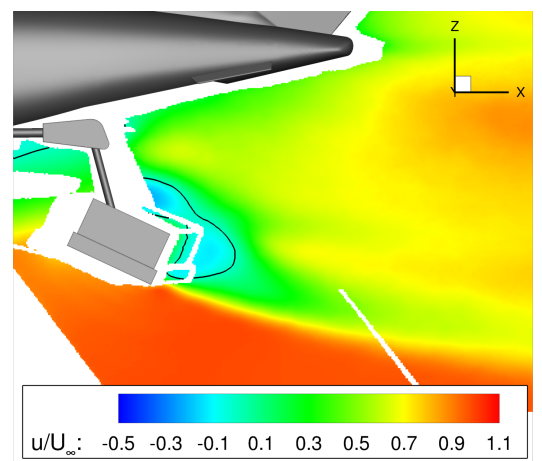

(f) PIV

Figure 9. Absolute $x$-velocity component $u$ at $y=0 \mathrm{~mm}$ for Cases $C$ (top) and $D$ (bottom), normalized with the onflow velocity of $U_{\infty}=36 \mathrm{~m} / \mathrm{s}$. The black solid line represents the iso-line where $u=0$.

\section{IV.C. Influence of the Support Platform}

Finally, the influence of the support platform is investigated by comparing the results of Cases $C$ and $D$. Figure 9 shows the $x$-velocity contour plots at the symmetry plane for the cases with (bottom) and without (top) support platform. For reference, the corresponding PIV results are depicted as well. Considering configurational aspects differences occur in predicting the region of detached flow around the cargo in Case $C$, which can easily be recognized by comparing the black solid iso-lines in Figures 9(a) - 9(f). The SA model in Figure 9(a) shows two regions in which a reversal of the $x$-velocity takes place. The first one starts at the upper edge of the front face and covers the entire upper surface. The second starts at the lower edge of the bottom surface and covers parts of the rear face of the cargo. The SST model, in contrast, predicts one continuous region of reversed flow as depicted in Figure 9(b). An embedded region can also be distinguished in that case, around the upper aft edge of the cargo. In principle, both turbulence models show the same physical behaviour, i. e. one vortex departing at the front face and two vortices with opposite rotational direction at the rear face. The PIV image in Fig. 9(c) shows a smaller wake region above the cargo and no visible region of reversed flow behind the cargo. The latter, however, is probably hidden due to the masked region. Nevertheless, assuming it exists there as well, its size is considerably smaller than in CFD.

A good qualitative agreement can be observed in the remaining field, with slight deviations around the lower linkage bar. In that region, similar to what could be observed in the same region in Case B, the CFD simulations predict higher velocities than PIV. However, in this case, the velocity magnitude does not exceed the onflow velocity. The reason for the latter results from the geometric arrangement relative to the aircraft, which now differs significantly from Case $B$ as shown in Fig. 1. The cargo body is no longer immersed into the undisturbed flow field below the aircraft. Instead, the lower linkage bar is directly located in the wake of the open ramp. Hence, the flow velocity it faces is lower than the onflow velocity, prohibiting an acceleration beyond the onflow velocity of $36 \mathrm{~m} / \mathrm{s}$. Generally, increasing the geometric complexity by attaching the support platform below the cargo in Case $D$ leads to an enlarged wake region. As shown in Figures 9(d) and 9(e), now both turbulence models show a continuous region of reversed flow, extending downstream to nearly one length of the cargo body. Comparing the PIV results for both configurations, 


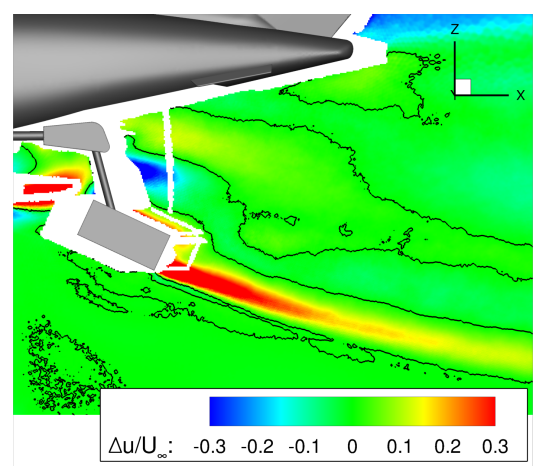

(a) PIV-SA

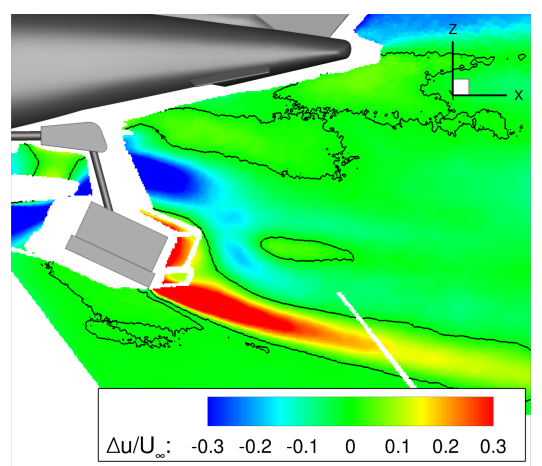

(d) PIV-SA

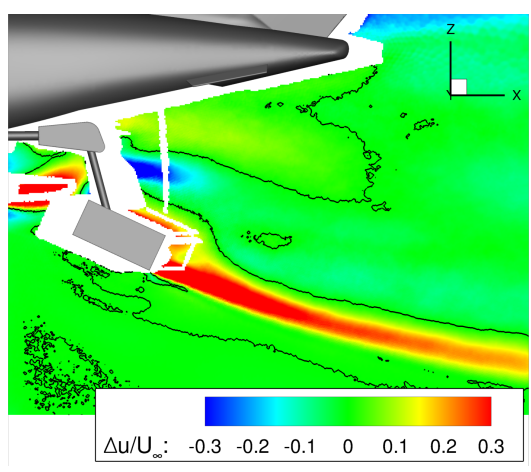

(b) PIV-SST

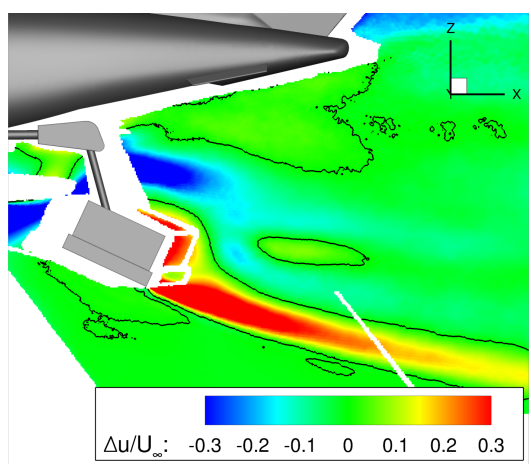

(e) PIV-SST

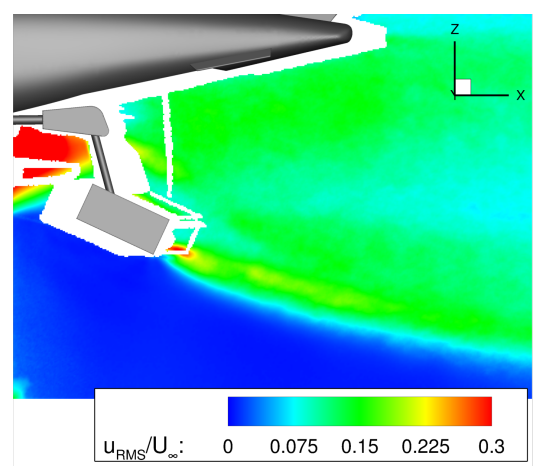

(c) PIV RMS

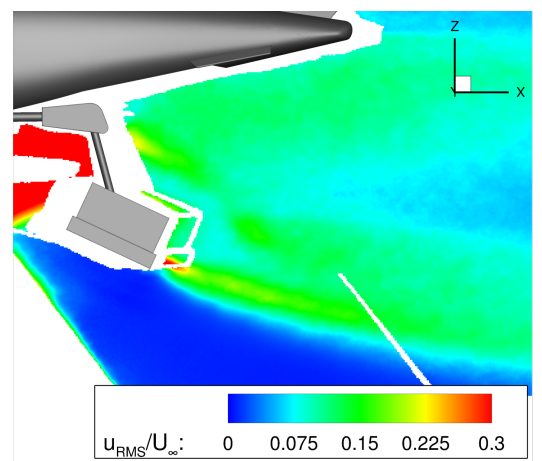

(f) PIV RMS

Figure 10. Relative $x$-velocity component $\Delta u$ at $y=0 \mathrm{~mm}$ for Cases $C$ (top) and $D$ (bottom), normalized with the onflow velocity of $U_{\infty}=36 \mathrm{~m} / \mathrm{s}$.

Figs. 9(c) and 9(f), the enlarged wake in Case $D$ is clearly visible there as well. In Case $C$, however, the PIV data shows no region of separated flow behind the cargo, whereas the simulations clearly do.

Fig. 10 reveals two regions of distinct differences between CFD and PIV: (i) the flow past the lower linkage bar and (ii) the shear layer from the lower side of the cargo. Regarding the first region, very high RMS values of the $x$-velocity can be observed between the lower linkage bar and the ramp of the aircraft as shown in Figs. 10(c) and 10(f). Thus, a highly unsteady flow behaviour in that region, probably due to the ramp vortices, adversely affects the CFD solution accuracy. In the second region, difficulties in correctly predicting the shear layer have already been identified in Case $B$ as discussed above. Moderate RMS values are present in that region, indicating a less pronounced flow unsteadiness. Hence, a grid adaption to more precisely resolve the shear layer would certainly reduce the gap to the experimental data.

In general, both turbulence models produce fairly identical results. Apart from the flow past the lower linkage bar, the results comply very well with the PIV data in qualitative terms. Quantitatively, the occurring deviations are of same order as discussed before at Case $B$.

\section{Conclusion}

This paper summarizes a validation study for different simplified airdrop configurations in static conditions. The generic cargo bodies were rigidly attached at different positions in the wake of a military transport aircraft with open ramp to assess the effects of flow interaction. A static positioning of the cargo bodies was a prerequisite to allow for a sufficient number of independent snapshots for a statistically reliable PIV evaluation. Nonetheless, the position of each body was not defined arbitrarily, but rather been derived from former experimental and numerical airdrop trajectory investigations carried out by DLR. The validation is based on an evaluation of field velocity data. The latter was numerically obtained by applying a steady RANS approach with four turbulence model of different fidelity, using unstructured meshes. In addition, mean and root mean square data based on three-component PIV measurements were used. Apart from the steady computations an exemplary unsteady computation was performed to assess the potential benefit of 
applying time-accurate methods in the present flow conditions being characterized by massive separation and vortex interaction.

To summarize the performance of the steady simulation for the aerodynamically complex cases, in particular the one with parachute, it can be stated that the steady approach is able to produce satisfying results in the sense that it very well resolves the prevailing flow features. This holds especially for the SA, the SST and the SARC turbulence models which produce largely identical results. Minor advantages could be observed for the SST model as it manages to additionally capture at least parts of the flow separation in very close agreement to PIV. The RSM model, in contrast, did not provide any noticeable benefit. On the contrary, it even led to the highest deviations compared to PIV. Due to its superior physical modelling of turbulence, however, the RSM should in principle improve the solution quality. The fact that it did not could be attributed to its rather poor convergence behaviour. This is also supported by the speckled velocity distribution obtained in the wakes of the bodies, where alternating velocity differences could be observed. Regarding the SARC model it produced nearly similar results compared to the original SA model. Thus, no noteworthy advantage was observed for the SARC in the present conditions.

Nonetheless, bearing in mind the highly challenging flow conditions examined in this study, the qualitative agreement between CFD and PIV is even better than initially expected. Some of the observed discrepancies between simulation and experiment can certainly be traced back to the partially unsteady flow behaviour, which cannot be adequately resolved by steady RANS methods. When it comes to absolute numbers, i. e. the correct prediction of size and extension of regions of reversed flow, the correct spatial position of shear layers and capturing the effects of vortex shedding or the correct velocity magnitude at a given position in the flow field, it appears questionable if more accurate results could be obtained with the chosen steady approach.

The gaps can certainly be reduced by applying a solution-dependent mesh adaption or in conducting a general grid convergence study in order to get an impression about the impact of the mesh resolution on the solution accuracy. Moreover, the authors believe that the application of time-accurate methods, be it uRANS or even (D)DES, is absolutely mandatory to achieve a significant improvement in quantitative overall compliance. The exemplarily conducted uRANS simulation already proved that the solution quality can be improved if the time-accuracy is accounted for. Finally, simulations and experiment were based on a different level of considered geometric details. Thus, reducing the geometric differences could eventually lead to an even better agreement between CFD and PIV.

\section{Acknowledgments}

The authors would like to thank the PIV group (Department Experimental Methods) of the DLR Institute of Aerodynamics and Flow Technology and the entire team of the DNW-NWB wind tunnel facility for their strong support in preparing and conducting the experimental campaigns.

\section{References}

\footnotetext{
${ }^{1}$ Roosenboom, E. W. M., Schade, N., Acisu, I., and Schröder, A., "Experimental and Numerical Investigation of the Near-Field Wake behind a Transport Aircraft Configuration with an Open Ramp," Paper, $4^{\text {th }}$ Symposium on Integrating CFD and Experiments in Aerodynamics, von Karman Institute, Rhode-Saint-Genèse, Belgium, September 2009.

${ }^{2}$ Roberts, D. W. and Judy, A. D., "Separation Flight Tests of a Small Unmanned Air Vehicle from a C-130 Transport Aircraft," In Flight Test - Sharing Knowledge and Experience, Meeting Proceedings RTO-MP-SCI-162, Paper 19, pp. 19-119-12, Neuilly-sur-Seine, France, 2005.

${ }^{3}$ Benney, R. J., Krainski, W. J., Onckelinx, P., Delwarde, C., Mueller, L., and Vallance, M., "NATO Precision Airdrop Initiatives and Modeling and Simulations Needs," In Fluid Dynamics of Personnel and Equipment Precision Delivery from Military Platforms, Meeting Proceedings RTO-MP-AVT-133, Keynote 2, pp. KN2-1-KN2-22, Neuilly-sur-Seine, France, 2006.

${ }^{4}$ Serrano, M., Leigh, E., III, W. J., Forsythe, J. R., Morton, S. A., and Squires, K. D., "Computational Aerodynamics of the C-130 in Airdrop Configuration," AIAA Paper 2003-0229, 41 ${ }^{\text {st }}$ Aerospace Sciences Meeting and Exhibit, Reno, NV, USA, January 2003.

${ }^{5}$ Morton, S. A., Tomaro, R. F., and Noack, R. W., "An Overset Unstructured Grid Methodology Applied to a C-130 with a Cargo Pallet and Extraction Parachute," AIAA Paper 2006-0461, 44 ${ }^{\text {th }}$ AIAA Aerospace Sciences Meeting and Exhibit, Reno, NV, USA, January 2006.

${ }^{6}$ McQuilling, M. and Potvin, J., "Simulating the Flows About Cargo Containers Used During Parachute Airdrop Operations," AIAA Paper 2010-4565, $28^{\text {th }}$ AIAA Applied Aerodynamics Conference, Chicago, IL, USA, June 2010.

${ }^{7}$ Jann, T. and Geisbauer, S., "Approximated Steady Aerodynamic Characteristics for two Cuboids and a Hemispherical Shell used in Airdrop Simulation," AIAA Paper 2013-1282, $22^{\text {nd }}$ AIAA Aerodynamic Decelerator Systems Technology Conference and Co-located Conferences, Daytona Beach, FL, USA, March 2013.
} 
${ }^{8}$ Geisbauer, S., Schade, N., Enk, S., Schmidt, H., and Arnold, J., "Experimental and Numerical Investigation of the Flow Topology During Airdrop Operations," AIAA Paper 2011-2565, 21 ${ }^{\text {st }}$ AIAA Aerodynamic Decelerator Systems Technology Conference and Seminar, Dublin, Ireland, May 2011.

${ }^{9}$ Schade, N., "Simulation of Store Separation," New Results in Numerical and Experimental Fluid Mechanics VIII, edited by A. Dillmann, G. Heller, H.-P. Kreplin, W. Nitsche, and I. Peltzer, Notes on Numerical Fluid Mechanics and Multidisciplinary Design, Springer, 2011.

${ }^{10}$ Schade, N., "Simulation of Trajectories of Cuboid Cargos Released from a Generic Transport Aircraft," AIAA Paper 2011-3959, 29 ${ }^{\text {th }}$ AIAA Applied Aerodynamics Conference, Honolulu, HI, USA, June 2011.

${ }^{11}$ Desabrais, K. J., "The Motion and Aerodynamics of an Airdrop Platform," AIAA Paper 2004-4845, 22 ${ }^{\text {nd }}$ AIAA Applied Aerodynamics Conference and Exhibit, Portland, OR, USA, August 2004.

${ }^{12}$ Desabrais, K. J., "Aerodynamic Forces on an Airdrop Platform," AIAA Paper 2005-1634, $18^{\text {th }}$ AIAA Aerodynamic Decelerator Systems Technology Conference and Seminar, Munich, Germany, May 2005.

${ }^{13}$ Löser, T. and Bergmann, A., "Capabilities of Deployment Tests at DNW-NWB," In Fluid Dynamics of Personnel and Equipment Precision Delivery from Military Platforms, Meeting Proceedings RTO-MP-AVT-133, Paper 13, pp. 13-1-13-11, Neuilly-sur-Seine, France, 2006.

${ }^{14}$ Jann, T., "Coupled Simulation of Cargo Airdrop from a Generic Military Transport Aircraft," AIAA Paper 2011-2566, $21^{\text {st }}$ AIAA Aerodynamic Decelerator Systems Technology Conference and Seminar, Dublin, Ireland, May 2011.

${ }^{15}$ McQuilling, M. and Potvin, J., "Effect of the Transient Nature of Flow on Annular Parachute Drag Prediction," Journal of Aircraft, Vol. 49, No. 2, March-April 2012, pp. 566-575.

${ }^{16}$ Roosenboom, E., Schröder, A., Agocs, J., and Geisler, R., "Experimental investigation of the flow field topology for several cargo drop configurations," AIAA Paper 2012-3198, 28 ${ }^{\text {th }}$ AIAA Aerodynamic Measurement Technology, Ground Testing and Flight Testing Conference, New Orleans, LA, USA, June 2012.

${ }^{17}$ Bergmann, A., "Modern Wind Tunnel Techniques for Unsteady Testing - Development of Dynamic Testing Rigs," Hermann Schlichting - 100 Years, edited by R. R. et al., Vol. 102 of Notes on Numerical Fluid Mechanics and Multidisciplinary Design, Springer, 2009, pp. 59-77.

${ }^{18}$ Roosenboom, E., Schröder, A., Agocs, J., and Geisler, R., "Coherent Wake Structures for Transport Aircraft at Cargo Airdrop Configurations Including Parachutes," AIAA Paper submitted for publication, $31^{\text {st }}$ AIAA Applied Aerodynamics Conference, San Diego, CA, USA, June 2013.

${ }^{19}$ DNW - German-Dutch Wind Tunnels, "NWB," http://www.dnw.aero/windtunnels.aspx?id=290\&menuid=34\&subid=290, retrieved May 2013.

${ }^{20}$ Bergmann, A., "The Aeroacoustic Wind Tunnel DNW," AIAA Paper 2012-2173, $18^{\text {th }}$ AIAA/CEAS Aeroacoustics Conference, Colorado Springs, CO, USA, June 2012.

${ }^{21}$ CentaurSoft, "CENTAUR Software," http://www.centaursoft.com, retrieved May 2013.

${ }^{22}$ Gerhold, T., Galle, M., Friedrich, O., and Evans, J., "Calculation of complex three-dimensional configurations employing the DLR-tau-code," AIAA Paper 1997-0167, 35 th AIAA Aerospace Sciences Meeting and Exhibit, Reno, NV, USA, January 1997.

${ }^{23}$ Gerhold, T., "Overview of the Hybrid RANS Code TAU," MEGAFLOW - Numerical Flow Simulation for Aircraft Design, edited by N. Kroll and J. Fassbender, Vol. 89 of Notes on Numerical Fluid Mechanics and Multidisciplinary Design, Springer, 2005, pp. 81-92.

${ }^{24}$ Schwamborn, D., Gerhold, T., and Heinrich, R., "The DLR TAU-Code: Recent Applications in Research and Industry," In Proceedings of the European Conference on Computational Fluid Dynamics, ECCOMAS, CFD 2006, edited by P. Wesseling, E. Oñate, and J. Périaux, The Netherlands, 2006.

${ }^{25}$ Kroll, N., Rossow, C.-C., Schwamborn, D., and Thiele, F., "MEGAFLOW - A Numerical Flow Simulation System," Paper ICAS 1998-2.7.4, $21^{\text {st }}$ International Congress of the Aeronautical Sciences (ICAS), Melbourne, Australia, September 1998.

${ }^{26}$ Kroll, N., Rossow, C.-C., Schwamborn, D., Becker, K., and Heller, G., "MEGAFLOW - A Numerical Flow Simulation Tool for Transport Aircraft Design," Paper ICAS 2002-1.10.5, $23^{\text {rd }}$ International Congress of the Aeronautical Sciences (ICAS), Toronto, Canada, September 2002.

${ }^{27}$ Spalart, P. and Allmaras, S., "A One-Equation Turbulence Model for Aerodynamic Flows," AIAA Paper 1992-439, 30 ${ }^{t h}$ Aerospace Sciences Meeting and Exhibit, Reno, NV, USA, January 1992.

${ }^{28}$ Menter, F. R., "Two-Equation Eddy-Viscosity Turbulence Models for Engineering Applications," AIAA Journal, Vol. 32, No. 8, August 1994, pp. 1598-1605.

${ }^{29}$ Spalart, P. and Shur, M., "On the Sensitization of Turbulence Models to Rotation and Curvature," Aerospace Science and Technology, Vol. 1, No. 5, August 1997, pp. 297-302.

${ }^{30}$ Zhong, L., "Effect of RANS Turbulence Models on Computation of Vortical Flow over Wing-Body Configurations," Transactions of the Japan Society for Aeronautical and Space Sciences, Vol. 48, No. 161, November 2005, pp. $152-160$.

${ }^{31}$ Eisfeld, B., "Numerical Simulation of Aerodynamic Problems with the SSG/LRR- $\omega$ Reynolds Stress Turbulence Model Using the Unstructured TAU Code," New Results in Numerical and Experimental Fluid Mechanics VI, edited by C. Tropea, S. Jakirlic, and H. Heinemann, Vol. 96 of Notes on Numerical Fluid Mechanics and Multidisciplinary Design, Springer, Heidelberg, 2007, pp. 356-363.

${ }^{32}$ Brodersen, O., Eisfeld, B., Raddatz, J., and Frohnapfel, P., "DLR Results from the Third AIAA CFD Drag Prediction Workshop," Journal of Aircraft, Vol. 45, No. 3, May-June 2008, pp. 823-836.

${ }^{33}$ Brodersen, O., Crippa, S., Eisfeld, B., Keye, S., and Geisbauer, S., "DLR Results from the Fourth AIAA CFD Drag Prediction Workshop," AIAA Paper 2010-4223, $28^{t h}$ AIAA Applied Aerodynamics Conference, Chicago, IL, USA, June 2010.

${ }^{34}$ Geisbauer, S., "Numerical Spoiler Wake Investigations at the Borders of the Flight Envelope," AIAA Paper 2011-3811, $29^{\text {th }}$ AIAA Applied Aerodynamics Conference, Honolulu, HI, USA, June 2011. 
${ }^{35}$ Jameson, A., Schmidt, W., and Turkel, E., "Numerical Solution of the Euler Equations by Finite Volume Methods using Runge-Kutta Time Stepping Schemes," AIAA Paper 81-1259, $14^{\text {th }}$ AIAA Fluid and Plasma Dynamics Conference, Palo Alto, CA, USA, June 1981. 